\title{
An unified approach to meteorological modelling based on multiple-scales asymptotics
}

\author{
R. Klein \\ FB Mathematik \& Informatik, Freie Universität Berlin, Arnimallee 2-6, 14195 Berlin, Germany \\ Received: 23 October 2007 - Revised: 22 February 2008 - Accepted: 25 February 2008 - Published: 18 March 2008
}

\begin{abstract}
In 2003, the author suggested a mathematical framework for the derivation of reduced meteorological models at a Mathematics conference (5th ICIAM, Sydney, Australia), (Klein, 2004). The framework consists of (i) nondimensionalization of the 3-D compressible flow equations on the rotating sphere, (ii) identification of universal nondimensional parameters, (iii) distinguished limits between these and additional problem-specific parameters, and (iv) multiple scales expansions in the remaining small parameter $\epsilon$. This parameter may be interpreted as the cubic root of the centripetal acceleration due to the Earth's rotation divided by the acceleration of gravity, see also Keller (1951), Eq. (10). For the mojority of reduced models of theoretical meteorology that we have come across, the approach allowed us to generate systematic derivations starting directly from the 3$\mathrm{D}$ compressible flow equations on the rotating sphere. The framework's potential fully shows in multiscale interaction studies such as Klein (2006), in which we incorporated bulk microphysics closures for moist processes and derived scale interaction models for deep convection. Currently, we study the structure, evolution, and motion of Hurricane strength H1/H2 vortices (Mikusky, 2007), large-scale stratocumulus cloud decks, and planetary-synoptic scale interaction models which should be relevant for Earth System Models of Intermediate Complexity (EMICs). Here we summarize the general framework and use the example of quasi-geostrophic theory to demonstrate its application.
\end{abstract}

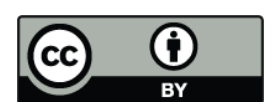

Correspondence to: R. Klein

(rupert.klein@zib.de)

\section{Introduction}

One important aim of theoretical meteorology is the development of simplified model equations that selectively describe some specific phenomena out of the large variety of scaledependent processes observed in atmospheric flows. The common practice of meteorological scale analysis proceeds as follows:

One first identifies a basic equation system that is considered adequate to represent all the processes in detail that are participating in the phenomenon of interest. Depending on the application at hand, this basic system could be the full compressible flow equations, an anelastic or incompressible Boussinesq system, or the hydrostatic primitive equations. Next one assesses from observations or simulations typical length and time scales, and often also typical amplitudes of variations of the flow variables associated with the phenomenon of interest. Using the chosen lengths, times, and amplitudes in scaling all terms in the basic equation system, one then decides which terms will dominate others, i.e., which terms may be neglected w.r.t. others in a reduced model. Often, these three steps do not yield a closed set of equations, and in this case advanced mathematical analysis of, e.g., higher-order perturbation equations is necessary in order to close the system.

While this procedure has proven extremely useful, it has, in the author's view, two considerable disadvantages. First, one arrives at a reduced model through a combination of physical and mathematical arguments, the corroboration of which generally requires advanced meteorological expertise and physical intuition besides mathematical skills. This is unsatisfactory in the context of teaching, where one would like to build the theories of meteorology systematically from a common baseline, which most will agree should be the set of compressible flow equations on a rotating sphere.

Published by Copernicus Publications on behalf of the European Geosciences Union. 
Table 1. Universal characteristics of atmospheric motions.

\begin{tabular}{ll}
\hline Earth's radius & $a=6 \times 10^{6} \mathrm{~m}$ \\
Earth's rotation rate & $\Omega \sim 10^{-4} \mathrm{~s}^{-1}$ \\
Acceleration of gravity & $g=9.81 \mathrm{~ms}^{-2}$ \\
Sea level pressure & $p_{\text {ref }}=10^{5} \mathrm{~Pa}$ \\
$\mathrm{H}_{2} \mathrm{O}$ freezing temp. & $T_{\text {ref }} \sim 273 \mathrm{~K}$ \\
Equator-pole temp. diff. & $\left.\Delta T\right|_{\mathrm{p}} ^{\mathrm{eq}} \sim 50 \mathrm{~K}$ \\
Dry gas constant & $R=287 \mathrm{~ms}^{-2} / \mathrm{K}$ \\
Dry isentropic exponent & $\gamma=1.4$ \\
\hline
\end{tabular}

Second, there is a wide range of modern applied mathematics techniques for multiscale analysis, stochastics, and scientific computing whose potential for advances in meteorological modelling is only beginning to be explored today. A mathematically transparent roadmap showing how existing reduced models of theoretical meteorology relate to the full compressible flow equations would support attempts at building related interdisciplinary bridges - its absence will likely hamper progress in this direction.

The present paper summarizes the author's current understanding of what such a roadmap could look like. The general approach was proposed in (Klein, 2004) and it has led to or been an important part of several recent new developments, see Majda and Klein (2003); Klein et al. (2004); Majda and Biello (2004); Mikusky et al. (2005); Biello and Majda (2006); Klein and Majda (2006); Majda (2007a,b); Mikusky (2007), and references therein.

Notably, Keller and Ting (1951) already anticipated the foundations of the present approach in an internal report of the Institute for Mathematics and Mechanics of New York University (today's Courant Institute).

The interested reader may also want to consult Zeytounian (1990) and the rich collection of references therein which provide detailed derivations of reduced models for a wide range of atmospheric flows using methods of singular perturbation analysis. The present work has been greatly stimulated by this volume.

Before getting involved with the essence of this paper, we should be quite explicit about its scope and limitations. The multiple scales asymptotics approach proposed here does allow us to reproduce very systematically a wide range of well-known meteorological model equations, and it paves the way for an equally systematic study of scale interactions. Once a flow regime of interest has been identified using physical arguments and intuition, the present approach carves out its mathematical essence and strips the derivation of appropriate reduced model equations of the overhead of physical argumentation. It does not work the other way round, however: there are infinitely many asymptotic expansion schemes which will yield reduced equations that are meaningless in the sense that they do not describe any phenomenon that one will ever observe in the Earth's atmo- sphere. As Pedlosky (1987) put it in the preface to the first edition of Geophysical Fluid Dynamics, "The union of physical and intuitive reasoning with mathematical analysis forms the central theme". The present work is an attempt to add further clarity on the mathematical side of this coin.

Section 2 will explicitly demonstrate that there are a few universal dimensionless parameters characterizing atmospheric flows that are independent of any specific flow phenomenon. Two of these parameters are small and motivate asymptotic analyses. We will emphasize the importance of distinguished limits, propose a universal one applicable to atmospheric flows, and then introduce the general multiplescales asymptotic expansion scheme that is the centerpiece of our approach. Section 3 provides a re-derivation of the quasi-geostrophic theory as a showcase. We end with a few conclusions.

\section{Universal parameters, distinguished limits, and non- dimensionalization}

Here we summarize the basic scaling arguments that justify a unified approach to the derivation of reduced models for atmospheric flows based on multiple scales asymptotic techniques. To elucidate our main points, we restrict the discussion here to inviscid compressible flows on a rotating sphere. Diabatic effects, such as radiation, water phase transitions, or turbulent transport will be represented as lumped terms in the governing equations to be specified later. Extensions of the framework to include moist processes have been developed recently by Klein and Majda (2006).

\subsection{Universal parameters and distinguished limits}

Table 1 displays several physical variables that are characteristic of atmospheric flows, and that are valid independently of the typical length and time scales of any specific flow phenomenon:

The mean sea level pressure $p_{\text {ref }}$ is set by the requirement that it balance the weight of a vertical column of air. Thus, it is directly given by the total mass of the atmosphere which, to a very good approximation, is evenly distributed over the sphere. A reference temperature $T_{\text {ref }}$ is set roughly by the global radiation balance which, even without greenhouse gases, would render the mean near-surface air temperature near $250 \mathrm{~K}$. The actual value in Table 1 is the freezing temperature of water under standard conditions, i.e., $T_{\text {ref }} \sim 273 \mathrm{~K}$, which is about midway between the observed maximal and minimal near-surface air temperatures. The pole-to-equator air temperature difference near the surface, $\left.\Delta T\right|_{\mathrm{p}} ^{\mathrm{eq}}$, is a consequence of the latitudinal variation of the sun's irradiation and meridional transport processes due to atmospheric motions. The order of magnitude of this temperature difference may be considered long-term stable and is used as a reference quantity here. The dry air gas constant, $R$, and isentropic 
exponent, $\gamma$, as thermodynamic properties of air are also universally characteristic for atmospheric flows, because their variations due to admixtures of water vapor, trace gases, and the like are no larger than a few percent in general.

Based on these eight basic reference quantities, four independent dimensionless combinations can be composed. To define combinations with intuitive interpretations, we introduce as auxiliary quantities the pressure scale height, $h_{\mathrm{sc}}$, the characteristic speed $c_{\text {ref }}$ of barotropic gravity waves, and a reference density, $\varrho_{\text {ref }}$, via

$$
\begin{aligned}
& h_{\mathrm{sc}}=p_{\text {ref }} /\left(g \rho_{\text {ref }}\right) \sim 10 \mathrm{~km} \\
& c_{\mathrm{ref}}=\sqrt{g h_{\mathrm{sc}}} \sim 300 \mathrm{~ms}^{-1} \\
& \rho_{\text {ref }}=p_{\text {ref }} /\left(R T_{\text {ref }}\right) \sim 1 \mathrm{~kg} \mathrm{~m}^{-3} .
\end{aligned}
$$

Then we let

$$
\begin{aligned}
& \Pi_{1}=\frac{h_{\mathrm{sc}}}{a} \sim 1.67 \cdot 10^{-3}, \\
& \Pi_{2}=\frac{\left.\Delta T\right|_{\mathrm{p}} ^{\mathrm{eq}}}{T_{\mathrm{ref}}} \sim 0.18, \\
& \Pi_{3}=\frac{c_{\mathrm{ref}}}{\Omega a} \sim 0.5 .
\end{aligned}
$$

The interpretations of $\Pi_{1}$ and $\Pi_{2}$ should be obvious, while the parameter $\Pi_{3}$ compares a typical barotropic, i.e., external gravity wave speed with the tangential speed of points on the equator as induced by Earth's rotation.

Remark: The sound speed, $\sqrt{\gamma p_{\text {ref }} / \rho_{\text {ref }}}$ is comparable to the external gravity wave speed, $c_{\mathrm{ref}}=\sqrt{g h_{\mathrm{sc}}}$ according to Eq. (1).

The parameter $\Pi_{1}$ is definitely quite small. $\Pi_{2}$ is not extremely small, yet, many successful developments in theoretical meteorology have relied on scale analysis (asymptotics) in terms of, e.g., Rossby numbers or internal wave Froude numbers with values in a similar range. Finally, for $\Pi_{3}$ one may argue that, even though it is less than unity, one may be hard pressed to consider it "asymptotically small." Deviating somewhat from our earlier work cited above, we will consider $\Pi_{3} \ll 1$ in the present notes.

There is little hope for success with asymptotic expansions that would allow $\Pi_{1}, \Pi_{2}$, and $\Pi_{3}$ to vary independently in a limit process: even for the simple example of a linear oscillator with small mass and small damping such an expansion in two independent parameters does not to exist!

Remark: If it would, the limit of vanishing mass and damping would be unique. Yet, if one lets the mass vanish much faster than the damping, the resulting motion is non-oscillatory and purely damped. If, in turn, the damping vanishes sufficiently much faster than the mass, then the limit solution is highly oscillatory with slow decay of the oscillation amplitude.

Thus, for the present parameters, we need to adopt a distinguished (or coupled) limit, and we investigate the following scaling relationships below,

$$
\Pi_{1} \sim \varepsilon^{3}, \quad \Pi_{2} \sim \varepsilon, \quad \Pi_{3} \sim \sqrt{\varepsilon}, \quad \text { as } \quad \varepsilon \rightarrow 0 .
$$

These limits are compatible with the estimates in Eq. (2) for actual values of $\varepsilon \sim 1 / 8 \ldots 1 / 6$. We will adopt $\varepsilon$ as the reference expansion parameter for asymptotic analyses below. Any additional small or large non-dimensional parameter that may be associated with singular perturbations in the governing equations is subsequently tied to $\varepsilon$ through suitable further distinguished limits.

Remark: Keller and Ting (1951) already proposed to use the acceleration ratio, $\varepsilon \sim\left(a \Omega^{2} / g\right)^{1 / 3}=\left(\Pi_{1} / \Pi_{3}^{2}\right)^{1 / 3}$, as a basic expansion parameter for meteorological modelling. When $\Pi_{3}=\mathcal{O}$ (1), this is equivalent to Eq. (3) above.

Remark: In contrast to Eq. (3), in the present author's earlier work the scaling $\Pi_{3}=\mathcal{O}(1)$ was adopted. A slightly modified limit regime is introduced here because it appears to unify our current developments of planetary balanced models with Pedlosky's derivations of the quasi-geostrophic theory in (Pedlosky, 1987), see also Sect. 3.

\subsection{Nondimensionalization and multiple scales expansions}

With $p_{\text {ref }}$ and $T_{\text {ref }}$, and through the ideal gas equation of state, $\rho=p / R T$, Table 1 immediately suggests reference values for the nondimensionalization of pressure, temperature, and density. But what about velocity, length, and time?

\subsubsection{Hydrostatic - geostrophic velocity scale}

Most theories for atmospheric flows rely on the assumption that typical flow speeds are small compared with the speed of barotropic gravity waves $c_{\text {ref }}$ which, except for a factor of $\sqrt{\gamma}$, matches the speed of sound. Here and in the rest of this section we make this assumption explicit by introducing a reference speed

$$
u_{\mathrm{ref}}=\frac{g h_{\mathrm{sc}}}{\Omega a} \frac{\left.\Delta T\right|_{\mathrm{p}} ^{\mathrm{eq}}}{T_{\mathrm{ref}}}=c_{\mathrm{ref}} \Pi_{2} \Pi_{3} \sim \varepsilon^{\frac{3}{2}} c_{\mathrm{ref}}
$$

for the nondimensionalization of the flow velocity. A typcial value is $u_{\text {ref }}=25 \mathrm{~m} / \mathrm{s}$. The reader may verify that the above expression has in fact the dimension of a velocity, but what is the motivation for this choice? We will resolve this question later when we rederive the quasi-geostrophic theory (QG). (See also the Remark at the end of this section and Eq. (18).) 


\subsubsection{Classical dimensionless parameters}

The selection of a velocity scale in Eq. (4) allows us to express several classical non-dimensional parameters of theoretical meteorology (and fluid dynamics) in terms of our small parameter. The (barotropic) Froude and Mach numbers become

$$
\overline{\mathrm{Fr}}=\frac{\mathrm{M}}{\sqrt{\gamma}}=\frac{u_{\mathrm{ref}}}{c_{\mathrm{ref}}} \sim \varepsilon^{\frac{3}{2}} .
$$

For the Froude number based on the internal gravity wave speed, $c_{\text {int }}=\sqrt{g h_{\mathrm{sc}} \frac{\left.\Delta \Theta\right|_{z=0} ^{h_{\mathrm{sc}}}}{\Theta_{\mathrm{ref}}}} \sim c_{\text {ref }} \sqrt{\varepsilon}$ we have

$$
\mathrm{Fr}=\frac{u_{\mathrm{ref}}}{c_{\mathrm{int}}}=\frac{\overline{\mathrm{Fr}}}{\sqrt{\varepsilon}} \sim \varepsilon .
$$

Here we took into account that the potential temperature difference across the troposphere roughly matches the pole-toequator temperature difference, so that

$$
\left.\left.\Delta \Theta\right|_{z=0} ^{h_{\mathrm{sc}}} \sim \Delta T\right|_{\mathrm{p}} ^{\mathrm{eq}} .
$$

The Rossby number based on the pressure scale height, $h_{\mathrm{sc}}$, reads

$$
\operatorname{Ro}_{h_{\mathrm{sc}}}=\frac{u_{\mathrm{ref}}}{h_{\mathrm{sc}} \Omega}=\frac{c_{\mathrm{ref}} \Pi_{2} \Pi_{3}}{\Omega a} \frac{a}{h_{\mathrm{sc}}}=\frac{\Pi_{2} \Pi_{3}^{2}}{\Pi_{1}} \sim \frac{1}{\varepsilon} .
$$

The internal Rossby radius, $L$, is defined as the characteristic length that an internal gravity wave would travel during the Earth's rotation time, i.e., $L \sim c_{\text {int }} / \Omega$. The associated Rossby number scales as

$$
\mathrm{Ro}=\frac{u_{\mathrm{ref}}}{L \Omega}=\Pi_{2} \Pi_{3} \frac{c_{\mathrm{ref}}}{c_{\text {int }}}=\frac{\Pi_{2} \Pi_{3}^{2}}{\sqrt{\varepsilon}} \sim \varepsilon .
$$

Analogously, the Oboukhov (or external Rossby) radius, $L_{\mathrm{Ob}}$, scales as

$$
L_{\mathrm{Ob}} \sim \frac{c_{\mathrm{ref}}}{\Omega}=L \frac{c_{\mathrm{ref}}}{c_{\mathrm{int}}} \sim \frac{L}{\sqrt{\varepsilon}},
$$

and it comes with the Oboukhov-scale (or external) Rossby number

$$
\mathrm{RoOb} \sim \varepsilon^{\frac{3}{2}} .
$$

Notice also that

$$
\frac{L}{\sqrt{\varepsilon}} \sim L_{\mathrm{Ob}} \sim \sqrt{\varepsilon} a \quad \text { where } \quad a \sim \frac{h_{\mathrm{sc}}}{\varepsilon^{3}} .
$$

Thus, with the present distinguished limits for $\Pi_{1}, \Pi_{2}, \Pi_{3}$, and $\varepsilon$ the Oboukhov (or external Rossby) radius is asymptotically bracketed by the internal Rossby radius, $L$, and the planetary scale, $a$, from below and above, respectively (see also Pedlosky, 1987, Eq. 6.5.23f).

\subsubsection{Space and time scalings}

As we are interested in multiple scales problems and will consistently account for different characteristic lengths of specific phenomena in our analyses through appropriate coordinate scalings, the particular choice of reference length and time scales for non-dimensionalization should not make any difference in the end. We opt here to use, $h_{\mathrm{sc}}$, i.e., the smallest length scale that suggests itself just from the fundamental parameters in Table 1 via Eq. (1), to nondimensionalize all lengths. The associated advection time serves as a reference time. Thus,

$$
\ell_{\mathrm{ref}}=h_{\mathrm{sc}} \quad \text { and } \quad t_{\mathrm{ref}}=\frac{h_{\mathrm{sc}}}{u_{\mathrm{ref}}} .
$$

2.3 Scaled governing equations and general multiple scales expansion scheme

With these scalings, the nondimensional governing equations in the rotating Earth system may be written as

$$
\begin{aligned}
\frac{D \boldsymbol{v}_{\|}}{D t}+\varepsilon 2(\boldsymbol{\Omega} \times \boldsymbol{v})_{\|}+\frac{1}{\varepsilon^{3}} \frac{1}{\rho} \nabla_{\|} p & =\boldsymbol{Q}_{\boldsymbol{v}_{\|}}, \\
\frac{D w}{D t}+\varepsilon 2(\boldsymbol{\Omega} \times \boldsymbol{v})_{\perp}+\frac{1}{\varepsilon^{3}} \frac{1}{\rho} p_{z} & =Q_{w}-\frac{1}{\varepsilon^{3}}, \\
\frac{D \rho}{D t}+\rho \nabla \cdot \boldsymbol{v} & =0, \\
\frac{D \Theta}{D t} & =Q_{\Theta},
\end{aligned}
$$

where

$$
\Theta=\frac{p^{1 / \gamma}}{\rho}
$$

is the dimensionless potential temperature, with $p, \rho$ the pressure and density, respectively, $\boldsymbol{v}_{\|}$is the horizontal and $w$ the vertical velocity, $\boldsymbol{\Omega}$ is the vector of Earth's rotation, and, due to the chosen nondimensionalization, the term $-1 / \varepsilon^{3}$ in the vertical momentum balance denotes the influence of gravity. The terms $\boldsymbol{Q}_{\boldsymbol{v}}, Q_{p}$, and $Q_{\Theta}$ represent additional effects which in a concrete application may stem from turbulence closures or similar models for the net influence of non-resolved scales. Also,

$$
\frac{D}{D t}=\partial_{t}+\boldsymbol{v}_{\|} \cdot \nabla_{\|}+w \partial_{z}
$$

is the time derivative along particle trajectories.

Klein (2004) suggested to consider the small parameter $\varepsilon$ as introduced above as the general singular asymptotic expansion parameter for theoretical developments in meteorology (although adopting a slightly different distinguished limit for $\Pi_{3}$ from Eq. (2). To this end, the solution vector $\mathcal{U}=\left(p, \Theta, \boldsymbol{v}_{\|}, w\right)^{t}$ is expanded in powers of $\varepsilon$ (or some fractional power thereof), and all expansion functions are allowed to depend on a collection of space-time coordinates 
that are scaled again by powers of $\varepsilon$. The most straightforward version of such a scheme reads

$$
\begin{aligned}
& \mathcal{U}(\boldsymbol{x}, z, t, ; \varepsilon)= \\
& \sum_{i} \varepsilon^{i} \mathcal{U}^{(i)}\left(\ldots, \frac{t}{\varepsilon}, t, \varepsilon t, \ldots, \frac{\boldsymbol{x}}{\varepsilon}, \boldsymbol{x}, \varepsilon \boldsymbol{x}, \ldots \frac{z}{\varepsilon}, z, \ldots\right) .
\end{aligned}
$$

In practical applications it might be necessary to work with fractional powers of $\varepsilon$ for the scaling of the coordinates, or more general asymptotic sequences, $\phi^{(i)}(\varepsilon)$, (see, e.g., Majda and Klein, 2003; Mikusky et al., 2005; Mikusky, 2007).

In a number of publications, we have demonstrated that a wide range of known simplified model equations of theoretical meteorology can be rederived in a unified fashion starting from the full compressible flow equations in (14) and suitable specializations of the multi-scale ansatz in Eq. (17). To derive a typical existing model, one would retain one scaled time, one scaled vertical coordinate, and one pair of scaled horizontal coordinates, respectively. The successful re-derivation of an established reduced model for some specific class of phenomena may then be considered as a "validation" of the general approach. We will demonstrate the formal procedures in the next chapter. Successful examples in question are the re-derivations of anelastic models and the weak temperature gradient approximation for convective scale motions in (Botta et al., 1999, 2000; Klein, 2000); the Matsuno-Gill model for synoptic-scale flows in the tropics and the equations for the planetary tropical wave guide in (Majda and Klein, 2003); the semi-geostrophic theory in (Klein, 2004), Ekman boundary layer theory in (Klein et al., 2004), and of Eliassen's balanced vortex model in (Mikusky et al., 2005; Mikusky, 2007).

Notice that most of the references cited go beyond the mentioned re-derivations of existing models to consider interactions across multiple scales or related issues in the design of numerical methods. See also Majda and Biello (2004); Biello and Majda (2006); Klein and Majda (2006); Majda (2007a) for further advanced developments.

Figure 1 summarizes the range of length and time scales that can be accessed by coordinate scalings in terms of powers of the universal asymptotic expansion parameter, $\varepsilon$, from Eq. (3). Three diagonals of this scaling grid can be identified with advective (principal diagonal), internal wave (first subdiagonal), and acoustic wave motions (second sub-diagonal).

Remark: The particular choice of a reference velocity in Eq. (4) does in no way restrict our degrees of freedom in constructing simplified asymptotic models. If, for example, we were to consider flows that are inherently compressible, so that systematically $|\boldsymbol{v}| \sim c_{\mathrm{re}}$, then the asymptotic expansion scheme for the (dimensionless) flow velocity would read

$$
\boldsymbol{v}=\frac{1}{\varepsilon^{\frac{3}{2}}} \sum_{i=0} \varepsilon^{i} \boldsymbol{v}^{(i)}\left(\ldots, \boldsymbol{x}, \varepsilon \boldsymbol{x}, \ldots \frac{z}{\varepsilon}, z, \ldots t, \varepsilon t, \ldots\right) .
$$

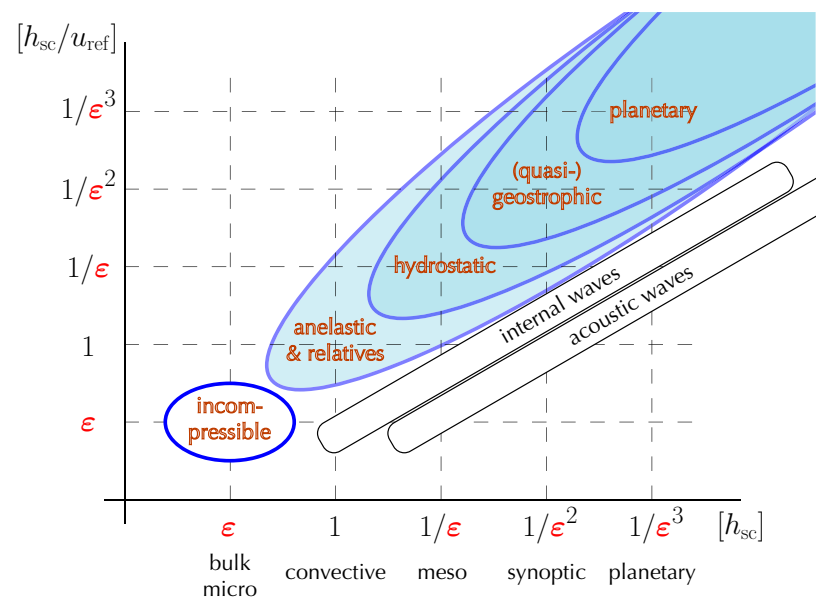

Fig. 1. Range of meteorological length and time scales that can be accessed through coordinate scalings in terms of powers of the universal expansion parameter $\varepsilon$ from Eq. (3). Advective, internal wave, and acoustic wave processes are associated with the principal, first sub-, and second sub-diagonal, respectively, in this diagram. See Eq. (12) for a characterization of the Oboukhov scale (or external Rossby radius) which lies in between the synoptic and planetary scales in this diagram.

\section{Derivation of the quasi-geostrophic (QG) theory}

Here we employ our general asymptotics-based approach from the previous section to rederive the classical quasigeostrophic model (see, e.g., Pedlosky, 1987). To provide a largely self-contained derivation we will re-iterate several findings already formulated in the last section.

\subsection{Asymptotic expansion scheme}

For the derivation of this theory, we will take the dimensionless form of the compressible flow equations from Eq. (14) as our point of departure. For simplicity of the exposition we assume adiabatic flow, dropping the various source terms here.

The quasi-geostrophic theory is designed to address flows on length scales comparable to the internal Rossby radius, and on time scales corresponding to horizontal advection across such distances. How can we access these length and time scales within our multiple scales asymptotic scheme?

The internal Rossby radius is defined as the distance which an internal gravity wave would travel during a characteristic Earth rotation time. This is equivalent to requiring

$$
L_{\mathrm{Ro}}=\frac{N h_{\mathrm{sc}}}{\Omega},
$$

where

$$
N=\sqrt{\frac{g}{\Theta} \frac{\partial \Theta}{\partial z^{\prime}}}
$$




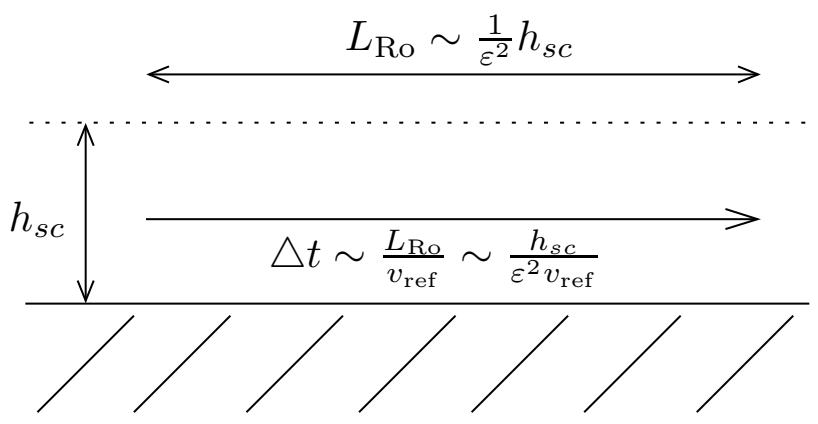

Fig. 2. Length and time scales addressed by quasi-geostrophic theory.

is the so-called Brunt-Väisälä or buoyancy frequency, and

$$
N h_{\mathrm{sc}}=\sqrt{g h_{\mathrm{sc}}} \sqrt{\frac{h_{\mathrm{sc}}}{\Theta} \frac{\partial \Theta}{\partial z^{\prime}}}=c_{\mathrm{ref}} \sqrt{\frac{h_{\mathrm{sc}}}{\Theta} \frac{\partial \Theta}{\partial z^{\prime}}}
$$

is a typical travelling speed of internal gravity waves. The reader may want to consult the established literature for corroboration.

Remark: Here and below, primes denote dimensional variables!

Remark: Another interpretation of the internal Rossby radius considers it the characteristic distance which an internal gravity wave would have to travel to become affected by the Coriolis effect.

Non-dimensionalizing $L_{\text {Ro }}$ by our reference length, $\ell_{\text {ref }}=h_{\text {sc }}$, and using the above we find

$$
\begin{aligned}
& \frac{L_{\mathrm{Ro}}}{h_{\mathrm{sc}}}=\frac{N}{\Omega}=\frac{c_{\mathrm{ref}}}{\Omega h_{\mathrm{sc}}} \sqrt{\frac{h_{\mathrm{sc}}}{\Theta} \frac{\partial \Theta}{\partial z^{\prime}}} \sim \frac{c_{\mathrm{ref}}}{\Omega a} \frac{a}{h_{\mathrm{sc}}} \sqrt{\frac{\left.\Delta T\right|_{\mathrm{p}} ^{\mathrm{eq}}}{T_{\mathrm{ref}}}} \\
& =\Pi_{3} \Pi_{1} \sqrt{\Pi_{2}}=\mathcal{O}\left(\varepsilon^{\frac{1}{2}} \frac{1}{\varepsilon^{3}} \varepsilon^{\frac{1}{2}}\right)=\mathcal{O}\left(\frac{1}{\varepsilon^{2}}\right) .
\end{aligned}
$$

Here we have used the scalings of our fundamental parameters from Table 1 as discussed in Eq. (1)-(3), and the (well established) observation that the equator-to-pole temperature differences are comparable to the vertical potential temperature variations across the troposphere, so that

$$
\frac{h_{\mathrm{sc}}}{\Theta} \frac{\partial \Theta}{\partial z} \sim \frac{\left.\Delta T\right|_{\mathrm{p}} ^{\mathrm{eq}}}{T_{\mathrm{ref}}} .
$$

With the estimate in Eq. (22), if we want to describe horizontal variations on scales comparable to $L_{\mathrm{Ro}}$, we should use the dimensionless horizontal coordinate

$$
\boldsymbol{\xi}=\frac{\boldsymbol{x}^{\prime}}{L_{\mathrm{Ro}}}=\frac{h_{\mathrm{sc}}}{L_{\mathrm{Ro}}} \frac{\boldsymbol{x}^{\prime}}{h_{\mathrm{sc}}}=\varepsilon^{2} \boldsymbol{x} .
$$

We will be interested here in phenomena associated with advection over distances of $L_{\text {Ro }}$, so we will use the time variable

$$
\tau=\frac{t^{\prime}}{L_{\mathrm{Ro}} / u_{\mathrm{ref}}}=\frac{h_{\mathrm{sc}}}{L_{\mathrm{Ro}}} \frac{t^{\prime}}{h_{\mathrm{sc}} / u_{\mathrm{ref}}}=\varepsilon^{2} t
$$

Finally, in order to study phenomena which occupy the full depth of the troposphere, we will use a vertical coordinate non-dimensionalized by the pressure scale height, $h_{\mathrm{sc}}$, i.e., we use our original dimensionless coordinate

$$
z=\frac{z^{\prime}}{h_{\mathrm{sc}}}
$$

These scalings are summarized in Fig. 2.

Our asymptotic expansion scheme for the solution written in terms of non-dimensional variables will thus read

$$
\mathcal{U}(t, \boldsymbol{x}, z ; \varepsilon)=\sum_{i} \varepsilon^{i} \mathcal{U}^{(i)}\left(\varepsilon^{2} t, \varepsilon^{2} \boldsymbol{x}, z\right),
$$

where

$$
\mathcal{U}=\left(p, \Theta, \boldsymbol{v}_{11}, w\right)^{t} .
$$

This is the announced specialization of the general multiple scales expansion scheme in Eq. (17) adapted to resolve advection phenomena on the length scale of the internal Rossby radius.

\subsection{Some preliminaries}

When inserting this expansion into the governing equations in Eq. (14) (without the source terms) we will have to account for the following transformation rules for the partial derivatives,

$$
\left.\frac{\partial}{\partial t}\right|_{\boldsymbol{x}, z ; \varepsilon}=\left.\varepsilon^{2} \frac{\partial}{\partial \tau}\right|_{\xi, z ; \varepsilon},\left.\quad \nabla_{\boldsymbol{x}}\right|_{t, z ; \varepsilon}=\left.\varepsilon^{2} \nabla_{\boldsymbol{\xi}}\right|_{\tau, z ; \varepsilon} .
$$

Here the subscripts indicate which variables are to be held constant when carrying out the partial differentiations.

We also anticipate the following properties of the background stratification of the atmosphere in order to save us some tedious calculations:

$$
\begin{aligned}
& \varrho(t, \boldsymbol{x}, z ; \varepsilon)=\varrho_{0}(z)+\varepsilon \varrho_{1}(z)+\varepsilon^{2} \varrho^{(2)}(\tau, \boldsymbol{\xi}, z)+\mathcal{O}\left(\varepsilon^{2}\right), \\
& p(t, \boldsymbol{x}, z ; \varepsilon)=p_{0}(z)+\varepsilon p_{1}(z)+\varepsilon^{2} p^{(2)}(\tau, \boldsymbol{\xi}, z)+\mathcal{O}\left(\varepsilon^{2}\right), \\
& \Theta(t, \boldsymbol{x}, z ; \varepsilon)=1+\varepsilon \Theta_{1}(z)+\varepsilon^{2} \Theta^{(2)}(\tau, \boldsymbol{\xi}, z)+\mathcal{O}\left(\varepsilon^{2}\right), \\
& w(t, \boldsymbol{x}, z ; \varepsilon)=\varepsilon^{3} w^{(3)}(\tau, \boldsymbol{\xi}, z)+\mathcal{O}\left(\varepsilon^{3}\right) .
\end{aligned}
$$

Remark: That the leading-and first-order thermodynamic variables are independent of time and do not vary horizontally can actually be derived within the present framework rather than having to be anticipated for appropriate initial data. The same is true for the vanishing of the leading three orders of vertical velocity, $w^{(0)}, w^{(1)}, w^{(2)}$.

Remark: The leading-order potential temperature must be a constant because of the order-of-magnitude analyses 
of the previous subsection which restrict variations of potential temperature to $\left.\Delta \Theta\right|_{z=0} ^{h_{\mathrm{sc}}} / T_{\text {ref }}=\mathcal{O}(\varepsilon)$. We may set this leading-order constant to $\Theta^{(0)} \equiv 1$ by choosing an appropriate reference temperature.

Remark: In Sect. 3.3.5 below we provide a brief derivation of the vertical velocity scaling in Eq. (30).

\subsection{Expansions of the governing equations}

The next steps are standard procedure. We insert the expansion scheme, collect like powers of $\varepsilon$, and separately equate the sum of these terms to zero, so as to create the usual hierarchy of perturbation equations.

\subsubsection{Mass conservation}

Expanding the mass conservation law, Eq. $\left(14_{3}\right)$, we find

$$
\begin{array}{rlrl}
\mathcal{O}\left(\varepsilon^{0}\right): & \varrho_{0} \nabla_{\xi} \cdot \boldsymbol{v}_{\|}^{(0)} & =0, \\
\mathcal{O}(\varepsilon) & : & \varrho_{0} \nabla_{\xi} \cdot \boldsymbol{v}_{\|}^{(1)}+\frac{\partial}{\partial z}\left(\varrho_{0} w^{(3)}\right) & =0 .
\end{array}
$$

In writing down the terms of $\mathcal{O}(\varepsilon)$ we have already neglected $\varrho^{(1)} \nabla_{\xi} \cdot \boldsymbol{v}_{\|}^{(0)}$ on account of Eq. $\left(31_{1}\right)$.

\subsubsection{Horizontal momentum balance}

\section{Splitting the Coriolis term}

Before expanding the momentum balances, we need to explicitly split the Coriolis term into its horizontal and vertical components, viz.

$$
\begin{aligned}
\boldsymbol{\Omega} \times \boldsymbol{v} & =\left(\boldsymbol{\Omega}_{\|}+\boldsymbol{k} \Omega_{\perp}\right) \times\left(\boldsymbol{v}_{\|}+\boldsymbol{k} w\right) \\
& =\underbrace{\left(\boldsymbol{\Omega}_{\|} \times \boldsymbol{v}_{\|}\right)}_{=(\boldsymbol{\Omega} \times \boldsymbol{v})_{\perp}}+\underbrace{\left(\Omega_{\perp} \boldsymbol{k} \times \boldsymbol{v}_{\|}+w \boldsymbol{\Omega}_{\|} \times \boldsymbol{k}\right)}_{=(\boldsymbol{\Omega} \times \boldsymbol{v})_{\|}} \\
& +\underbrace{\left(\Omega_{\perp} w \boldsymbol{k} \times \boldsymbol{k}\right)}_{=0, \text { as } \boldsymbol{k} \times \boldsymbol{k}=0} .
\end{aligned}
$$

We will need the vertical component of $\boldsymbol{\Omega}$ (see Fig. 3), which we expand as

$$
\begin{aligned}
\Omega_{\perp} & =\boldsymbol{k} \cdot \boldsymbol{\Omega} \\
& =\left(\boldsymbol{e}_{e q} \cos \vartheta+\boldsymbol{e}_{\boldsymbol{\Omega}} \sin \vartheta\right) \cdot \boldsymbol{e}_{\boldsymbol{\Omega}}|\boldsymbol{\Omega}| \\
& =|\boldsymbol{\Omega}| \sin \vartheta \\
& =|\boldsymbol{\Omega}| \sin \left(\vartheta_{0}+\frac{y^{\prime}}{a}\right) \\
& =|\boldsymbol{\Omega}| \sin \left(\vartheta_{0}+\varepsilon \xi_{2}\right) \\
& =\underbrace{|\boldsymbol{\Omega}| \sin \left(\vartheta_{0}\right)}_{=: \Omega_{0}}+\varepsilon \underbrace{|\boldsymbol{\Omega}| \cos \left(\vartheta_{0}\right)}_{=: \beta} \xi_{2}+\mathcal{O}(\varepsilon) \\
& =\Omega_{0}+\varepsilon \beta \xi_{2}+\mathcal{O}(\varepsilon) .
\end{aligned}
$$

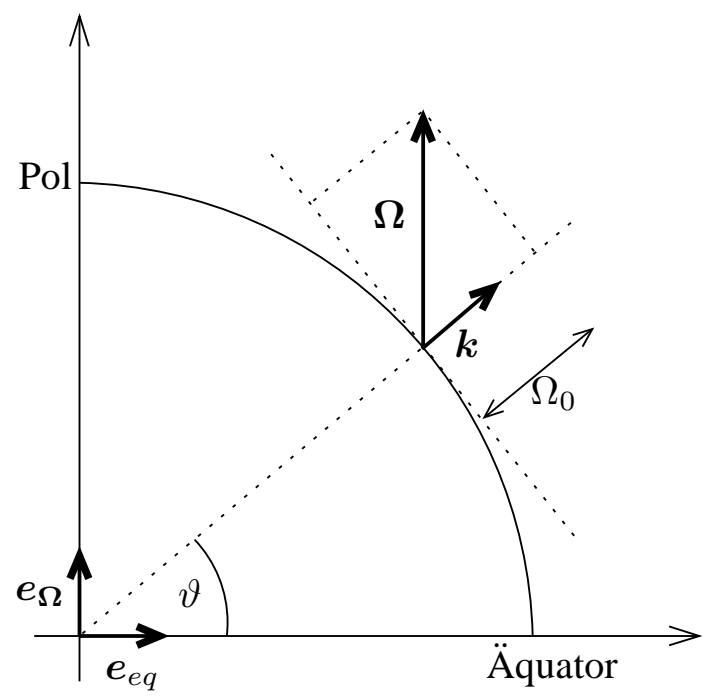

Fig. 3. Splitting of the coriolis term into a horizontal and a vertical component.

Here we have taken into account that $\vartheta$ is the arclength along a longitudinal circle divided by the radius of the reference sphere, $a$, introduced deviations from a reference latitude, so that $\vartheta=\vartheta_{0}+y^{\prime} / a$, and recalled that $h_{\text {sc }} / a=\varepsilon^{3}$ and $\xi_{2}=\varepsilon^{2} y^{\prime} / h_{\text {sc }}$. The rest is Taylor expansion of the sine function about the reference latitude.

Since $w^{(i)} \equiv 0$ for $i \in\{0,1,2\}$, we also know that $w \boldsymbol{\Omega}_{\|} \times \boldsymbol{k}=\mathcal{O}(\varepsilon)$, i.e., it is "little-oh" of $\varepsilon$ and thus vanishing faster than $\varepsilon$ as $\varepsilon \rightarrow 0$. In summary, we find

$$
\begin{aligned}
& (\boldsymbol{\Omega} \times \boldsymbol{v})_{\|}=\left(\Omega_{0}+\varepsilon \beta \xi_{2}\right) \boldsymbol{k} \times \boldsymbol{v}_{\|}+\mathcal{O}(\varepsilon), \\
& (\boldsymbol{\Omega} \times \boldsymbol{v})_{\perp}=\boldsymbol{\Omega}_{\|} \times \boldsymbol{v}_{\|} .
\end{aligned}
$$

\subsubsection{Expansion of the horizontal momentum balance}

The horizontal momentum balance, written in terms of the new variables, $(\tau, \boldsymbol{\xi}, z)$, reads

$$
\boldsymbol{v}_{\| \tau}+\left(\boldsymbol{v}_{\|} \cdot \nabla_{\xi}\right) \boldsymbol{v}_{\|}+\frac{1}{\varepsilon^{2}} w \boldsymbol{v}_{\| z}+\frac{1}{\varepsilon^{3}} \frac{\nabla_{\xi} p}{\varrho}+\frac{1}{\varepsilon}(\hat{\boldsymbol{\Omega}} \times \boldsymbol{v})_{\|}=0 .
$$

Using $w^{(i)} \equiv 0$ for $i \in\{0,1,2\}$, and that $p^{(0)} \equiv p_{0}(z)$ and $p^{(1)} \equiv p_{1}(z)$, we may move on to the equation at $\mathcal{O}\left(\varepsilon^{-1}\right)$ where we find the geostrophic balance,

$$
\Omega_{0} \boldsymbol{k} \times \boldsymbol{v}_{\|}^{(0)}+\nabla_{\xi} \pi^{(2)}=0, \quad \text { with } \quad \pi^{(2)}=\frac{p^{(2)}}{\varrho_{0}},
$$

i.e., the balance of the horizontal Coriolis and pressure gradient forces. Geostrophic balance implies that, at leading order, the horizontal flow direction is perpendicular to the horizontal pressure gradient.

We verify for later purposes that

$$
\nabla_{\xi} \cdot \boldsymbol{v}_{\|}^{(0)}=0 \quad \text { and } \quad \boldsymbol{v}_{\|}^{(0)}=\frac{1}{\Omega_{0}} \boldsymbol{k} \times \nabla_{\xi} \pi^{(2)}
$$


by, respectively, applying $\left(\boldsymbol{k} \cdot\left(\nabla_{\xi} \times[\cdot]\right)\right)$ and $(\boldsymbol{k} \times[\cdot])$ to Eq. (36).

\subsubsection{Vertical momentum balance}

From the vertical momentum balance we obtain at orders $\varepsilon^{-5}$ to $\varepsilon^{-2}$

$$
\frac{\partial p^{(i)}}{\partial z}=-\varrho^{(i)} \quad(i=0,1,2,3) .
$$

Expanding the defining equation for the potential temperature, i.e., $\varrho \Theta=p^{\frac{1}{\gamma}}$ into

$$
\begin{array}{crl}
\mathcal{O}\left(\varepsilon^{0}\right): & \varrho_{0}=p_{0}{ }^{\frac{1}{\gamma}} \\
\mathcal{O}(\varepsilon): & \varrho_{1}+\varrho_{0} \Theta_{1}=p_{0}^{\frac{1}{\gamma}} \frac{p_{1}}{\gamma p_{0}} \\
\mathcal{O}\left(\varepsilon^{2}\right): & \varrho^{(2)}+\varrho_{1} \Theta_{1}+\varrho_{0} \Theta^{(2)}= \\
& & p_{0} \frac{1}{\gamma}\left(\frac{p^{(2)}}{\gamma p_{0}}+\frac{(1-\gamma) p_{1}^{2}}{2 \gamma^{2} p_{0}^{2}}\right)
\end{array}
$$

we obtain from Eq. (38)

$$
p^{(0)}-\frac{1}{\gamma} \frac{\partial p^{(0)}}{\partial z}=-1
$$

with exact solution

$$
p_{0}(z)=\left(1-\frac{\gamma-1}{\gamma} z\right)^{\frac{\gamma}{\gamma-1}} .
$$

In a similar way one solves the first order equation explicitly for given $\Theta_{1}(z)$. The details are not essential for the sequel, and the calculations are straightforward.

\subsubsection{Evolution of the potential temperature}

The first non-trivial asymptotic equation is extracted from the potential temperature transport equation at $\mathcal{O}\left(\varepsilon^{4}\right)$, and it reads

$$
\left(\frac{\partial}{\partial \tau}+\boldsymbol{v}_{\|}^{(0)} \cdot \nabla_{\xi}\right) \Theta^{(2)}+w^{(3)} \frac{d \Theta_{1}}{d z}=0 .
$$

Remark: Suppose the vertical velocity was expanded as $w=\varepsilon^{2} w^{(2)}+\varepsilon^{3} w^{(3)}+\ldots$. Then potential temperature transport equation at $\mathcal{O}\left(\varepsilon^{3}\right)$ would read

$$
w^{(2)} \frac{d \Theta_{1}}{d z}=0,
$$

which corroborates the expansion scheme adopted in Eq. (30) above. Similar arguments can be made for expansions involving even larger vertical velocities.

\subsection{Summary of the leading-order balances}

Using the expansion scheme in Eq. (30), we have found first that the background state is in hydrostatic balance, i.e.,

$$
\frac{d p_{i}}{d z}=-\varrho_{i} \quad(i=0,1) .
$$

These equations can be integrated directly using Eq. (39) with $p_{0}(0)=1, p_{1}(0)=0$, and for given distribution $\Theta_{1}(z)$ of the first-order potential temperature.

The remaining primary unknowns for description of the flow field are then

$$
\left(\pi^{(2)}, \boldsymbol{v}_{\|}^{(0)}, w^{(3)}, \Theta^{(2)}\right)(\tau, \boldsymbol{\xi}, z),
$$

where $\pi^{(2)}=p^{(2)} / \varrho_{0}$, and they satisfy the following balance and transport equations:

$$
\begin{aligned}
& \frac{\text { Hydrostatic Balance }}{} \\
& \qquad \frac{\partial \pi^{(2)}}{\partial z}=\Theta^{(2)}
\end{aligned}
$$

\section{$\underline{\text { Geostrophic Balance }}$}

$$
\Omega_{0} \boldsymbol{k} \times \boldsymbol{v}_{\|}^{(0)}+\nabla_{\xi} \pi^{(2)}=0
$$

\section{$\underline{\text { Anelastic Constraint }}$}

$$
\varrho_{0} \nabla_{\xi} \cdot \boldsymbol{v}_{\|}^{(1)}+\frac{\partial}{\partial z}\left(\varrho_{0} w^{(3)}\right)=0
$$

\section{Potential Temperature Transport}

$$
\left(\frac{\partial}{\partial \tau}+\boldsymbol{v}_{\|}^{(0)} \cdot \nabla_{\xi}\right) \Theta^{(2)}+w^{(3)} \frac{d \Theta_{1}}{d z}=0
$$

If it were not for the appearance of the first-order divergence, $\nabla_{\xi} \cdot \boldsymbol{v}_{\|}^{(1)}$ in Eq. (48), we would have the same number of equations as we have unknowns. As it is, the system is as yet unclosed. In the next section, we will extract additional information on $\nabla_{\xi} \cdot \boldsymbol{v}_{11}^{(1)}$ from the next higher order horizontal momentum equation in the form of a solvability condition that may be interpreted as a vorticity transport equation,

\section{First-Order Solvability/Vorticity Transport}

$$
\left(\partial_{\tau}+\boldsymbol{v}_{\|}^{(0)} \cdot \nabla_{\xi}\right)\left(\zeta^{(0)}+\beta \xi_{2}\right)+\Omega_{0} \nabla_{\xi} \cdot \boldsymbol{v}_{\|}^{(1)}=0 .
$$

where,

$$
\zeta^{(0)}=\boldsymbol{k} \cdot\left(\nabla_{\xi} \times \boldsymbol{v}_{\| 1}^{(0)}\right),
$$


is the vorticity of the leading-order velocity field, and $\beta$ is the meridional derivative of the Coriolis parameter as defined in Eq. (33).

This completes the summary of the quasi-geostrophic model equations.

Remark: We have given the QG equations here in a somewhat unusual form that is as close as possible to that of the original equations. This way it remains transparent that Eq. (46) and Eq. (47) are direct consequences of the vertical and horizontal momentum balances, respectively, Eq. (48) emerges from mass conservation, and Eq. (49) from the potential temperature transport equation. These equations can all directly be read off the original equations at the appropriate orders in the asymptotic expansion.

The closure for $\nabla_{\xi} \cdot \boldsymbol{v}_{\|}^{(1)}$ in Eq. (50) emerges as a solvability condition at $\mathcal{O}\left(\varepsilon^{0}\right)$ in the horizontal momentum balance as will be shown in the next section.

Remark: The present summary of asymptotic limit equations for the quasi-geostrophic flow regime shows that restricting to large spacial and long temporal scales induces time independent constraints on the solutions. Instead of evolution equations for the primary unknowns in the compressible flow equations (the densities of mass, momentum, and energy) we find three such constraints or balances! Only the potential temperature evolution equation in Eq. (49) and the vorticity transport equation in Eq. (50) have maintained the original "prognostic" (time evolution) character.

These constraints imply that only if the initial data for a given flow problem satisfy the constraint, at least at the given orders, can we hope that the approximate asymptotic solution will remain close to the exact solution as time evolves.

Such changes of the mathematical type of the governing equations is an important characteristic of many singular perturbation problems.

3.5 First-order solvability condition / existence of $\nabla_{\xi} p^{(3)}$

Consider the scaled horizontal momentum balance from Eq. (35), which we had already written in terms of our new coordinates, $(\tau, \boldsymbol{\xi}, z)$, at $\mathcal{O}\left(\varepsilon^{0}\right)$,

$$
\begin{aligned}
\left(\boldsymbol{v}_{\|}^{(0)}\right)_{\tau} & +\left(\boldsymbol{v}_{\|}^{(0)} \cdot \nabla_{\xi}\right) \boldsymbol{v}_{\|}^{(0)}+\left[\frac{\nabla_{\xi} p}{\varrho}\right]^{(3)} \\
& +\Omega_{0} \boldsymbol{k} \times \boldsymbol{v}_{\|}^{(1)}+\beta \xi_{2} \boldsymbol{k} \times \boldsymbol{v}_{\|}^{(0)}=0 .
\end{aligned}
$$

Using the fact that $\varrho_{0}, \varrho_{1}$ depend on $z$ only, so that $\nabla_{\xi} \varrho_{0}=\nabla_{\xi} \varrho_{1}=0$, and $\pi^{(i)}=p^{(i)} / \varrho_{0}$ we rewrite the pressure gradient term as

$\left[\frac{\nabla_{\xi} p}{\varrho}\right]^{(3)}=\frac{1}{\varrho_{0}} \nabla_{\xi} p^{(3)}-\frac{\varrho_{1}}{\varrho_{0}^{2}} \nabla_{\xi} p^{(2)}=\nabla_{\xi} \pi^{(3)}-\nabla_{\xi}\left(\frac{\varrho_{1}}{\varrho_{0}} \pi^{(2)}\right)$.
Next we regroup Eq. (52) into first-order "geostrophic terms" on left-hand side and terms that distort the geostrophic balance, i.e., "ageostrophic terms", on the right,

$$
\begin{aligned}
& \nabla_{\xi} \pi^{(3)}+\Omega_{0} \boldsymbol{k} \times \boldsymbol{v}_{\|}^{(1)}= \\
& -\left(\frac{D^{(0)}}{D \tau} \boldsymbol{v}_{\|}^{(0)}+\beta \xi_{2} \boldsymbol{k} \times \boldsymbol{v}_{\|}^{(0)}-\nabla_{\xi}\left(\frac{\varrho_{1}}{\varrho_{0}} \pi^{(2)}\right)\right),
\end{aligned}
$$

where

$$
\frac{D^{(0)}}{D \tau}=\partial_{\tau}+\left(\boldsymbol{v}_{\|}^{(0)} \cdot \nabla_{\xi}\right) .
$$

We know that any gradient of a scalar is curl-free. In particular,

$$
\boldsymbol{k} \cdot\left(\nabla_{\xi} \times \nabla_{\xi} \phi\right) \equiv 0
$$

for any scalar function $\phi(\xi)$ that is sufficiently smooth. By applying the operator $\boldsymbol{k} \cdot\left(\nabla_{\xi} \times[\cdot]\right)$ to Eq. (54) we thus eliminate the terms involving $\pi^{(2)}$ and $\pi^{(3)}$. The remaining terms become

$$
\begin{aligned}
\boldsymbol{k} \cdot\left(\nabla_{\xi} \times\left(\Omega_{0} \boldsymbol{k} \times \boldsymbol{v}_{\|}^{(1)}\right)\right) & =\Omega_{0} \boldsymbol{k} \cdot\left(\boldsymbol{k}\left(\nabla_{\xi} \cdot \boldsymbol{v}_{\|}^{(1)}\right)\right) \\
& =\Omega_{0} \nabla_{\xi} \cdot \boldsymbol{v}_{\|}^{(1)}, \\
\boldsymbol{k} \cdot\left(\nabla_{\xi} \times\left(\boldsymbol{v}_{\|}^{(0)}\right)_{\tau}\right) & =\zeta_{\tau}^{(0)}, \\
\boldsymbol{k} \cdot\left(\nabla_{\xi} \times\left(\left(\boldsymbol{v}_{\|}^{(0)} \cdot \nabla_{\xi}\right) \boldsymbol{v}_{\|}^{(0)}\right)\right) & =\boldsymbol{v}_{\|}^{(0)} \cdot \nabla_{\xi} \zeta^{(0)}, \\
\boldsymbol{k} \cdot\left(\nabla_{\xi} \times\left(\beta \xi_{2} \boldsymbol{k} \times \boldsymbol{v}_{\|}^{(0)}\right)\right) & =\beta \boldsymbol{k} \cdot \boldsymbol{k}\left(\nabla_{\xi} \xi_{2} \cdot \boldsymbol{v}_{\|}^{(0)}\right) \\
& =\beta v^{(0)} \\
& =\beta \frac{D^{(0)}}{D \tau} \xi_{2} .
\end{aligned}
$$

Collecting, we find the vorticity transport equation,

$$
\frac{D^{(0)}}{D \tau}\left(\zeta^{(0)}+\beta \xi_{2}\right)+\Omega_{0} \nabla_{\xi} \cdot \boldsymbol{v}_{\| 1}^{(1)}=0,
$$

as announced in Eq. (50).

\subsection{Potential vorticity transport equation}

In Eqs. (47)-(50) we have taken care to display the quasigeostrophic balance equations in a form that reveals their close connection to the mass, momentum, and potential temperature evolution equations. This may not be the most practicable description in many applications, and it hides the central role of potential vorticity in the quasi-geostrophic regime.

In fact, one can rewrite Eq. (58) as a transport equation for the QG-potential vorticity,

$$
q=\zeta^{(0)}+\beta \xi_{2}+\frac{\Omega_{0}}{\varrho_{0}} \frac{\partial}{\partial z}\left(\frac{\varrho_{0} \Theta^{(2)}}{d \Theta_{1} / d z}\right),
$$


which then reads

$$
\frac{D^{(0)}}{D \tau} q=0 .
$$

Equipped with the additional constitutive relations

$$
\begin{aligned}
\boldsymbol{v}_{\|}^{(0)} & =-\frac{1}{\Omega_{0}} \boldsymbol{k} \times \nabla_{\xi} \pi^{(2)}, \\
\Theta^{(2)} & =\frac{\partial \pi^{(2)}}{\partial z}, \\
\zeta^{(0)} & =\boldsymbol{k} \cdot\left(\nabla_{\xi} \times \boldsymbol{v}_{\|}^{(0)}\right)=-\frac{1}{\Omega_{0}} \nabla_{\xi}^{2} \pi^{(2)}
\end{aligned}
$$

we have the QG theory in its classical form (Pedlosky, 1987). In fact, Eq. (60) describes advection of potential vorticity by the leading order velocity field $\boldsymbol{v}_{\|}^{(0)}$, which can be expressed in terms of the pressure gradient $\nabla_{\xi} \pi^{(2)}$ according to Eq. (61). Given the (advected) PV-field, one can retrieve the pressure field solving the ellliptic equation that results from inserting Eq. (61) into Eq. (59), viz.

$$
\nabla_{\xi}^{2} \pi^{(2)}+\frac{\Omega_{0}^{2}}{\varrho_{0}} \frac{\partial}{\partial z}\left(\frac{\varrho_{0}}{d \Theta_{1} / d z} \frac{\partial \pi^{(2)}}{\partial z}\right)=\Omega_{0}\left(q-\beta \xi_{2}\right) .
$$

\section{Conclusions}

This paper summarizes an approach to constructing reduced dynamical models for atmospheric motions that is (i) general enough to allow the re-derivation of a large set of well-known reduced models of theoretical meteorology, (ii) mathematically transparent, and that (iii) provides a sound basis for the description of multiple scales interactions.

In a first step we have identified eight universal physical characteristics of atmospheric motions and combined them to form four universal non-dimensional characteristic numbers, $\Pi_{1}, \Pi_{2}, \Pi_{3}$ from Eq. (2), and the dry air isentropic exponent, $\gamma$. Two of these parameters turned out to be quite small, motivating attempts at asymptotic analysis. The asymptotics of the simple problem of a linear oscillator with small mass and small damping shows that asymptotic expansions in multiple independent parameters generally yield non-unique results. This motivated the introduction of distinguished limits among the four atmospheric flow parameters, so that they were all tied to one remaining asymptotic expansion parameter, $\varepsilon$. Due to the chosen distinguished limits, $\varepsilon$ may equally be interpreted as the Rossby number for synoptic scale flows, as the cubic root of the atmosphere's global aspect ratio, as the cubic root of the square of a characteristic flow Mach number, etc..

Choosing a reference length comparable to the pressure scale height and an associated typical advection time to nondimensionalize the space and time coordinates, the derivation of classical reduced models for atmospheric flows proceeds as follows: Solutions to the full compressible flow equations are expanded in (not necessarily integer) powers of $\varepsilon$, with the expansion terms depending on asymptotically scaled space and time coordinates. For the example of the quasi-geostrophic theory (QG), this expansion reads

$$
\mathcal{U}(t, \boldsymbol{x}, z ; \varepsilon)=\sum_{i} \varepsilon^{i} \mathcal{U}^{(i)}\left(\varepsilon^{2} t, \varepsilon^{2} \boldsymbol{x}, z\right),
$$

with $\mathcal{U}$ denoting the vector of unknowns. The rest is standard singular perturbuation analysis. In Sect. 3 we have provided the detailed derivation of QG to demonstrate the application of the framework.

The present unified approach to meteorological modelling suggests itself immediately as a systematic framework for teaching purposes. It also lays the foundation for multiple scales analyses as documented in the references listed below. Recently, Majda (2007a) used the same line of argument to reveal how super-parameterizations (see, e.g., Grabowski, 2004) can be understood as heterogeneous multiscale methods (see E and Engquist, 2003). He discussed a wide range of separated length and time scales that are accessible through stretched space and time coordinates, $\left(\varepsilon^{i} \boldsymbol{x}, \varepsilon^{j} t\right)$, for various choices of $i, j$, and he derived coupling conditions between the scales consistent with multiple scales asymptotics.

It is worth noting that several of the key ideas outlined in the present report have already been around more than fifty years ago (Keller and Ting, 1951).

Acknowledgements. Part of the research that led to the concepts presented in this paper has been funded by Deutsche Forschungsgemeinschaft through grants KL 611/14, KL 611/15. The author thanks U. Harlander, M. Ehrendorfer, and A. Will for the opportunity to present this material at the general assembly of the European Geosciences Union, 2007.

Edited by: U. Harlander

Reviewed by: two anonymous referees

\section{References}

Biello, J. and Majda, A.: Transformations for temperature flux in multiscale models of the tropics, Theor. Comp. Fluid Dyn., 20, 405-420, 2006.

Botta, N., Klein, R., and Almgren, A.: Dry atmosphere asymptotics, PIK-Report 55, Potsdam Institute for Climate Impact Research, 1999.

Botta, N., Klein, R., and Almgren, A.: Asymptotic Analysis of a Dry Atmosphere, in: ENUMATH 99 - Proc. 3rd Europ. Conf. on Num. Math., Jyväskylä, Finland, edited by: Neittaanmäki, P. and Tiihonen, T., World Scientific, Singapore, 2000.

E, W. and Engquist, B.: The heterogeneous multiscale method, Comm. Math. Sci., 1, 87-132, 2003.

Grabowski, W.: An improved framework for superparameterization, J. Atmos. Sci., 61, 1940-1952, 2004.

Keller, J. and Ting, L.: Approximate equations for large scale atmospheric motions, Internal Report, Inst. for Mathematics 
\& Mechanics (renamed to Courant Institute of Mathematical Sciences in 1962), NYU, (http://www.arxiv.org/abs/physics/ 0606114), 1951.

Klein, R.: Asymptotic Analyses for Atmospheric Flows and the Construction of Asymptotically Adaptive Numerical Methods, ZAMM, 80, 765-777, 2000.

Klein, R.: An applied mathematical view of meteorological modelling, in: Applied Mathematics Entering the 21st century, Invited talks from the ICIAM 2003 Congress, Vol. 116, SIAM Proceedings in Applied Mathematics, 177-219, 2004.

Klein, R. and Majda, A.: Systematic multiscale models for deep convection on mesoscales, Theor. Comp. Fluid Dyn., 20, 525$551,2006$.

Klein, R., Mikusky, E., and Owinoh, A.: Multiple scales asymptotics for atmospheric flows, in: 4th European Conference of Mathematics, Stockholm, Sweden, edited by: Laptev, A., European Mathematical Society Publishing House, 201-220, 2004.

Majda, A.: Multiscale Models with Moisture and Systematic Strategies for Superparameterization, J. Atmos. Sci., 64, 2726-2734, 2007a.
Majda, A.: New multiscale models and self-similarity in tropical convection, J. Atmos. Sci., 64, 1393-1404, 2007 b.

Majda, A. and Biello, J.: A multiscale model for tropical intraseasonal oscillations, Proc. Natl. Acad. Sci. USA, 101, 4736-4741, 2004.

Majda, A. J. and Klein, R.: Systematic Multiscale Models for the Tropics, J. Atmos. Sci., 60, 393-408, 2003.

Mikusky, E.: On the structure of concentrated atmospheric vortices in the gradient wind regime and their motion on synoptic scales, Ph.D. thesis, Hamburg University, 2007.

Mikusky, E., Owinoh, A., and Klein, R.: On the influence of diabatic effects on the motion of 3D-mesoscale vortices within a baroclinic shear flow, in: Computational Fluid and Solid Mechanics 2005, Elsevier, Amsterdam, 766-768, 2005.

Pedlosky, J.: Geophysical Fluid Dynamics, Springer-Verlag, New York, 2. Edn., 1987.

Zeytounian, R.: Asymptotic modelling of atmospheric flows, Springer-Verlag, Berlin, Heidelberg, New York, 1990. 